\title{
Cognitive Behavioral Therapy for Insomnia in the Context of Cardiovascular Conditions
}

\author{
Samantha Conley $^{1} \cdot$ Nancy S. Redeker ${ }^{1}$
}

Published online: 10 July 2015

(C) Springer International Publishing AG 2015

\begin{abstract}
Cardiovascular disease (CVD) is the leading cause of death worldwide and is associated with high levels of morbidity, disability, and poor quality of life. The prevalence of insomnia symptoms and diagnosis of insomnia are high among those with CVD. Although insomnia appears to be important to CVD, less is known about the effects of insomnia treatment on important biological, sleep, symptom, quality of life, and functional and morbidity or mortality outcomes in people with or at risk for CVD. The purposes of this paper are to review the literature on the effects of CBT-I on insomnia, sleep, daytime symptoms, function, and biological outcomes in the context of CVD and to suggest implications for future research and practice. Limited available evidence suggests that CBT-I improves biomarkers that may contribute to CVD risk. CBT-I shows promise as a way to improve cardiovascular morbidity associated with the negative biological consequences of chronic insomnia, as well as symptom burden, functional performance, and quality of life in the large population of people who are living with chronic CVD and insomnia. Additional research is needed to answer basic questions about short- and long-term outcomes, the role of biomarkers, and optimal delivery methods of CBT-I in cardiac conditions.
\end{abstract}

This article is part of the Topical Collection on Insomnia - Behavioral Therapy

Nancy S. Redeker

Nancy.redeker@yale.edu

Samantha Conley

Samantha.conley@yale.edu

1 Yale School of Nursing, PO Box 27399, West Haven, CT 06516-7399, USA
Keywords Insomnia $\cdot$ Cardiovascular disease $\cdot$ Heart failure $\cdot$ Hypertension - Coronary heart disease $\cdot$ Hypertension . Fatigue $\cdot$ Cognitive behavioral therapy

\section{Introduction}

Cardiovascular conditions (CVD), such as hypertension, ischemic heart disease, stroke, and heart failure (HF), are the leading causes of death worldwide, accounting for over 17 million deaths per year [1] and high levels of morbidity, disability, and poor quality of life. Insomnia symptoms, including difficulty initiating or maintaining sleep and/or waking too early in the morning, accompanied by daytime dysfunction, occur in about $25-30 \%$ of adults [2, 3], and the disorder of chronic insomnia occurs in about $10 \%$ [4]. However, rates of insomnia symptoms and the diagnosis of insomnia are higher among those with CVD, including hypertension (25-44 \%) $[5,6]$ and acute coronary syndrome (37 \%) [7], and HF (51\%) [8], for example.

Emerging research suggests that insomnia, a disorder of arousal $[9,10]$, contributes to the development of cardiovascular conditions [11-14] independent of traditional risk factors [15]. Although data are mixed [16, 17], insomnia and short or long sleep duration [18-20] and insomnia with arousal were associated with hypertension [21]. Insomnia also predicted use of anti-hypertensive and other cardiovascular medications [22], coronary heart disease [23], and myocardial infarction $[24,25]$. Insomnia had a dose-related effect on incident HF and death [26], and may contribute to death from other cardiovascular conditions [14, 27], but data are conflicting $[15,28]$.

Among patients already diagnosed with chronic CVD, chronic insomnia is associated with disabling symptoms (e.g., fatigue, dyspnea, anxiety, depression, excessive daytime 
sleepiness, and pain in patients with HF) $[8,15,26,29-32]$ and was related to decrements in quality of life among patients in a cardiac rehabilitation program [33], patients with and without CVD in the Sleep Heart Health Study [34], and HF patients $[8,35]$. Symptoms, poor quality of life, and functional deficits are especially common, severe, and often intractable among patients with chronic CVD, even with evidence-based disease management. Although clinicians seldom consider the potential contributions of comorbid insomnia to these outcomes [36••], insomnia was closely associated with both self-reported and objective functional performance among HF patients $[8,26]$ and may contribute to poor quality of life in other CVD populations.

Although insomnia appears to be important to CVD, less is known about the effects of insomnia treatment on important biological, sleep, symptom, quality of life, and functional and morbidity or mortality outcomes in people with or at risk for CVD [37]. Cognitive behavioral therapy for insomnia (CBT-I) is a multimodal approach that includes cognitive (e.g., cognitive restructuring of dysfunctional beliefs and perceptions about sleep) and behavioral techniques (e.g., sleep restriction, stimulus control, and relaxation therapy) [38-40]. CBT-I has durable effects on insomnia, lacks the adverse consequences of hypnotic medications [41], and is acceptable to patients who often prefer it to hypnotic medications [42, 43]. The purposes of this paper are to review the literature on the effects of CBT-I on insomnia, sleep, daytime symptoms, function, and biological outcomes in the context of CVD and to suggest implications for future research and practice.

\section{Effects of CBT-I on Insomnia and Sleep Characteristics in People with CVD}

Several studies addressed the effects of CBT-I on comorbid insomnia among older adults with CVD and in studies that also included people with other medical diagnoses (osteoarthritis, chronic obstructive pulmonary disease) $[44,45 \cdot \bullet, 46$, 47]. CBT-I, compared with a self-management wellness program, improved self-reported sleep efficiency, latency, time awake after sleep onset, time in bed, number of naps, and sleep quality [46]. CBT-I also improved self-reported, but not actigraph-measured sleep characteristics [44], and there was no difference in outcomes between groups with specific medical comorbidities when compared with home-based audio relaxation training. Rybarczyk et al. [45••] compared two versions of a 6-week self-help CBT-I program (book and enhanced video, including video-taped CBT-I sessions) among 106 older adults with osteoarthritis, coronary artery disease, and those without major medical conditions (see Table 1 for study descriptions). Both approaches to CBT-I improved sleep, and the effects were maintained at 1 year (sleep efficiency $d=0.73$, sleep latency $d=0.53$, wake after sleep onset $d=$
0.64 , and total sleep time $d=0.62$ ). These effects are consistent with other studies conducted in general primary care populations and older adults (e.g., $[54,55])$ that presumably included some patients with CVD, but did not differentiate these diagnoses. While these studies $[44,45 \bullet \cdot, 46]$ included older adults with CVD, the samples were small, details about specific diagnoses, risk factors, and severity of CVD were not reported, and there was no focus on daytime sleep-related, symptoms important in CVD.

To our knowledge, only one CBT-I study was conducted in a group of patients selected entirely because they had CVD. Redeker et al. [36 ••, 51] conducted a pilot randomized controlled trial in which 48 patients with stable HF and at least mild chronic insomnia defined by the Insomnia Severity Index [56] were randomized to a group CBT-I intervention led by a psychologist $(n=29)$ or an attention control group consisting of HF self-management education and sleep hygiene education led by an advanced practice nurse $(n=19)$. Both conditions included four bi-weekly 1 -h group sessions over 8 weeks with telephone calls on intervening weeks. All participants had at least mild insomnia. Potential participants were excluded if they had untreated moderate-severe sleep apnea or unstable medical or psychiatric conditions. CBT-I, compared with the attention control condition, resulted in a moderate to large effect on insomnia severity $(d=.65, p=.03)$ and sleep quality $(d=.46, p=.14)$, after controlling for baseline insomnia and clinical and demographic characteristics. The CBT-I group experienced a mean decrease of more than 7 points on the insomnia severity index compared to the attention control group; $76 \%$ of participants in the CBT-I group improved across one or more levels of insomnia (severe to none) and no one deteriorated, compared with poorer outcomes in the attention control. The effect on sleep efficiency $(d=.70$, $p=.03$ ) was statistically large, with sleep efficiency increasing by $3.3 \%(\mathrm{SD}=6.15)$ in the CBT-I group and decreasing by $1.27 \%(\mathrm{SD}=7.20)$ in the attention control condition. Both groups had low levels of actigraph-recorded sleep efficiency at baseline (CBT-I, 72.6 $\pm 10.2 \%$; attention control, $77.3 \pm$ $7.1 \% ; p=$ ns) and follow-up (CBT-I, 75.9 $\pm 8.3 \%$; attention control, $76.1 \pm 11 \%$ ). While overall, the mean change in sleep efficiency may not be clinically significant, $15 \%$ of the population sample had an increase of $9.4 \%$ in sleep efficiency, a clinically meaningful increase. Notably, improvements in selfreported insomnia severity were maintained at 6 months after treatment. However, actigraph data were not obtained at this time period [51]. The results of this study, conducted with patients with stable HF and who received evidence-based HF management, were especially notable, given clinicians' frequent perceptions that sleep disturbance among HF is a primarily a consequence of poorly controlled HF. These findings are especially robust since the inclusion of symptom self-management information, adherence to medications, and sleep hygiene, in the attention control 
Table 1 CBT-I study description

\begin{tabular}{|c|c|c|c|}
\hline Authors (year) & Sample description & Study design & Intervention duration, and frequency \\
\hline $\begin{array}{l}\text { Irwin et al. }[48 \bullet \bullet] \\
\text { Irwin et al. }[49 \bullet] \\
\text { Carroll et al. }[50]\end{array}$ & $\begin{array}{l}N=123 \text { older adults (age } \geq 55) \text { with } \\
\text { chronic and primary insomnia }\end{array}$ & $\begin{array}{l}\text { RCT } \\
\text { CBT-I } \\
\text { Comparators: } \\
\text { Tai Chi Chih } \\
\text { Sleep Education } \\
(2: 2: 1)\end{array}$ & $\begin{array}{l}\text { Weekly } 120 \text {-min group meetings } \\
\text { over } 4 \text { months }\end{array}$ \\
\hline $\begin{array}{l}\text { Redeker et al. [51] } \\
\text { Redeker et al. [52] } \\
\text { Redeker et al. [53•] }\end{array}$ & $\begin{array}{l}N=48 \text { patients with stable NY Heart } \\
\text { Class I-III heart failure and insomnia }\end{array}$ & $\begin{array}{l}\text { RCT } \\
\text { CBT-I } \\
\text { Comparator: } \\
\text { Heart Failure Self-management } \\
\quad \text { Education }\end{array}$ & $\begin{array}{l}\text { 4-biweekly } 1 \mathrm{~h} \text { meetings with } \\
\text { 15-min phone calls on } \\
\text { intervening weeks }\end{array}$ \\
\hline Rybarczyk et al. [44] & $\begin{array}{l}N=38 \text { older adults (age } \geq 55) \text { with } 2 \\
\text { or more chronic illnesses and } \\
\text { comorbid insomnia }\end{array}$ & $\begin{array}{l}\text { RCT } \\
\text { CBT-I } \\
\text { Comparators: } \\
\text { Home-based audio relaxation } \\
\quad \text { treatment (HART) } \\
\text { Delayed-treatment }\end{array}$ & $\begin{array}{l}\text { CBT-I: } 8 \text {-weekly group classroom } \\
\text { sessions lasting } 1.5 \mathrm{~h} \\
\text { HART: } 7 \text { audiotaped recordings } \\
\text { used over } 6 \text { weeks lasting } 0.5 \\
\text { h with biweekly telephone class } \\
\text { lasting } 5 \text { min }\end{array}$ \\
\hline Rybarczyk et al. [46] & $\begin{array}{c}N=92 \text { older adults (age } \geq 55) \text { with } \\
\text { osteoarthritis, coronary artery } \\
\text { disease or pulmonary disease }\end{array}$ & $\begin{array}{l}\text { RCT } \\
\text { CBT-I } \\
\text { Comparators: } \\
\text { Stress management and wellness } \\
\quad \text { training }\end{array}$ & $\begin{array}{l}\text { 8-weekly classroom treatment } \\
\text { programs }\end{array}$ \\
\hline Rybarczyk et al. [45••] & $\begin{array}{l}N=106 \text { older adults (age } \geq 55) \text { with } \\
\text { osteoarthritis, coronary artery } \\
\text { disease or no major medical } \\
\text { conditions }\end{array}$ & $\begin{array}{l}\text { RCT } \\
\text { CBT-I: } \\
\text { Comparators: } \\
\text { Book format } \\
\text { Multi-media enhanced CBT-I }\end{array}$ & $\begin{array}{l}6 \text { modules with weekly phone } \\
\text { class }\end{array}$ \\
\hline
\end{tabular}

condition that might be expected to improve HF outcomes $[36 \bullet \bullet$.

\section{Effects of CBT-I on Daytime Symptoms, Functional Performance, and Other Health Outcomes}

Based on the evidence that insomnia [8], but not sleep disordered breathing, another common sleep disorder among CVD patients [57], was closely associated with daytime symptoms, including fatigue, depression, excessive daytime sleepiness, and both self-reported and objective measures of functional performance, Redeker and colleagues' (details reported above) also examined these outcomes [36••, 51].

CBT-I, compared to HF self-management education plus sleep hygiene, led to large improvements in fatigue $(d=.64$, $p=.04)$ and smaller improvements in self-reported physical function, measured with the Medical Outcomes SF-36 ( $d=.25, p=\mathrm{ns})$, but did not improve sleepiness, depression, or anxiety at short-term follow-up among 48 patients who had stable heart failure (HF).

In the same study, follow-up at 6 months revealed that fatigue continued to decrease $(d=1.23)$ in the CBT-I group, while increasing in the attention control group (HF selfmanagement education) [51], after controlling for clinical and demographic characteristics, baseline fatigue, and insomnia severity. There were improvements in anxiety and depression, with large effects on anxiety $(d=.69)$ and smaller effects on depression $(d=.13)$ over baseline at 6 months. Effects on anxiety and depression were both direct and indirect (through the effect on insomnia remission). These findings suggest the potential sustained effects of CBT-I on fatigue and mood.

Extending epidemiological findings that insomnia predicted incident HF and survival [26], Redeker et al. found that the estimated incidence rates of rehospitalization at 12 months, after controlling for baseline symptoms, were $13.2 \%$ among HF patients who received CBT-I, compared with $21.1 \%$ in the attention control group, after controlling for baseline symptoms and clinical and demographic characteristics $(\mathrm{OR}=0.32$, $\mathrm{CI}=0.04,2.46$ ) [51]. Nine percent of those with insomnia remission were rehospitalized, compared with $27 \%$ of those who did not have insomnia remission. The apparent differences in hospitalization between the CBT-I and the attention control group are consistent with outcomes of a study of CBT for depression, compared to usual care conducted with $42 \mathrm{HF}$ patients in which the researchers found improvements in cardiovascular event-free survival (mortality, cardiovascular hospitalizations, and emergency department visits), but no differences in depression follow-up in the CBT vs. usual care groups [58]. A larger study of CBT for stress management among participants $(n=362)$ who had experienced acute 
coronary events [59] demonstrated improvements in both event-free survival and reduced rates of ischemic cardiac events. Although sleep or insomnia were not evaluated in the latter two studies, it is possible that sleep improved in these groups. Given the interrelationships among insomnia and mood, and the importance of mood to quality of life and event-free survival, there is a need to further examine the generalized effects of CBT on mood and sleep, compared to the specific effects of CBT-I on mood and CVD outcomes.

\section{Biological Outcomes of CBT-I}

Insomnia and sleep loss are closely associated with high levels of psychological and physiological arousal [9] and activation of the HPA axis and sympathetic nervous system [9] that, in turn, may contribute to alterations in immunity and inflammation, important contributors to CVD risk [60]. Recent studies document associations between insomnia and inflammatory processes as measured by C-reactive protein [14, 61], autonomic dysfunction [10], and alterations in the HPA axis [62].

Evidence suggests that CBT-I may contribute to improved CVD outcomes through reductions in arousal and resulting improvements in inflammation. Three recent reports of a trial of CBT-I, compared with Tai Chi Chih (TCC), a "westernized" version of tai chi, and a sleep seminar control (SS), among older adults $[48 \bullet \bullet, 49 \bullet, 50]$ documented improvements in biomarkers that may contribute to reduced CVD risk and/or prevention of CVD exacerbation. Participants $(n=123)$ were aged 55 and older and had primary (DSM-IV-TR) [63] or general (International Classification of Sleep Disorders, Second edition) [64] insomnia. Exclusion criteria included other sleep disorders, use of hypnotics, and major depression, among others. Approximately $40-50 \%$ of study participants had comorbid medical conditions, of whom about $95 \%$ had CVD [50]. Participants were randomized to one of the three treatment conditions and followed for 16 months.

CBT-I compared with the other treatment conditions, improved risk of high CRP $(3.0 \mathrm{mg} / \mathrm{L})$ at 16 months, and insomnia remission was associated with reduced CRP at this time period [48••]. There were also CBT-I-related effects on composite metabolic and cardiovascular risk factors (i.e., highdensity lipoprotein, low-density lipoprotein, triglycerides, hemoglobin A1c, glucose, insulin, C-reactive protein, and fibrinogen) [50]. Both CBT-I and TCC reduced the chance of being in a high-risk metabolic group (defined as a multi-system risk score from the sum of the deviations across the cutoffs of the biomarkers) at 16 months, but only CBT-I improved the risk at 4 months. Improvements in sleep quality among participants with high-risk profiles at baseline led to classification in the lower risk group at 16 months. This research group also demonstrated that CBT-I reduced monocyte production of inflammatory cytokines (all $p<.05$ ) and pro-inflammatory gene expression across months $2,4,7$, and 16 of follow-up in the same sample as the earlier papers [61]. These findings also extend the findings of an earlier study of CBT-I, compared with a wait-list control group, conducted with breast cancer survivors [65] in which CBT-I led to increased secretion of IFN-gamma and lower increases in lymphocytes at posttreatment compared with control patients and provide beginning evidence that CBT-I may improve biomarkers of CVD risk and ultimately contribute to primary and secondary prevention of CVD among adults with chronic insomnia.

Using biological data from the 48 patients who participated in the CBT-I trial [53•], Redeker et al. [52] found that improvements in sleep quality and fatigue from baseline to post-CBT-I ( 2 weeks after completing 8 weeks of CBT-I or HF self-management education) were associated with increases in the ratio of daytime to nighttime urinary cortisol $[(r=0.29, p=.08)$ and $(r=0.54, p=.0007)]$, respectively. The ratio of daytime/nighttime urinary epinephrine was associated with wake after sleep onset (WASO) $(r=-0.52, p=.004)$. Although there was no statistically significant effect of CBT-I on cortisol ratio, these findings suggest the importance of CBT-I related improvements in insomnia and fatigue to the circadian rhythmicity of the HPA axis.

Taken together, the findings of the insomnia studies that have used measured biomarkers suggest the support of the potential benefits of CBT-I in preventing the development of CVD or its exacerbation through its metabolic, inflammatory, autonomic, and circadian effects. Further studies are needed to test that these effects are needed in a larger randomized controlled trials to determine the extent to which CBT-I reduces CVD morbidity and mortality.

\section{Discussion}

Limited available evidence suggests that CBT-I improves biomarkers that may contribute to CVD risk, insomnia, sleep characteristics, and daytime symptoms, among small groups of people with CVD who represent a large and diverse population, many of whom suffer from high symptom burden, poor quality of life, and functional decrements, as well as high levels of morbidity and mortality. One pilot randomized controlled trial among patients with HF, a group with especially high symptom burden, documented improvements in insomnia, large improvements in fatigue, and durable effects on anxiety and depression. These promising studies extend the large body of CBT-I research by focusing on the biological underpinnings of treatment effects, durable effects on daytime symptoms, and the potential condition-specific benefits of CBT-I for people with or at risk for CVD. The effects of the small group of studies reviewed are remarkable because they used attention control conditions with elements that might be expected to have some benefits for insomnia and sleep 
characteristics, in contrast with several past studies [66] in which the comparator was usual care or less robust modalities such as sleep hygiene, without controlling for the time with the therapist.

Although the studies reviewed are promising, continued study is needed to confirm and extend the findings. This may include mechanistic studies and larger, longitudinal randomized controlled RCTs to improve understanding of the sustained effects of CBT-I in HF, as well as other chronic cardiovascular conditions (e.g., ischemic heart disease, dysrhythmias). Suggestions for future research are discussed below and summarized in Table 2.

\section{CBT-I and Symptom Burden}

Redeker et al.'s study [52] suggests the benefits of CBT-I for reducing sleep-related symptoms among patients with $\mathrm{HF}$ and are similar to the findings of Fleming et al. [67] among cancer patients who often have high levels of fatigue, anxiety, and depression similar to CVD patients. The durable effects on daytime symptoms in HF are especially remarkable given the often-intractable nature of symptoms and suggest that the CBT-I recipients continue to use the skills learned after the conclusion of active treatment. Future studies should be conducted to address biobehavioral mechanisms of CBT-I, including factors that lead to continued use of CBT-I strategies and the biological outcomes of treatment efficacy. There is also a need to consider the effects of CBT-I on symptom clusters linked with poor sleep [31], given the multi-

Table 2 Suggestions for future research on the effects of insomnia and CBT-I in relation to cardiovascular disease

- Examine the factors that contribute to the sustained use of specific CBTI strategies to promote durability of treatment effects.

- Examine the biobehavioral mediators of the effects of CBT-I among CVD patients

- Evaluate the effects of specific components of CBT-I on CVD outcomes, including morbidity, quality of life, and health care resource utilization.

- Examine the long-term effects of CBT-I on sleep, daytime symptoms, and quality of life among a variety of CVD patients.

- Consider the extent to which aging, gender, and comorbidity moderate treatment effects.

- Examine the extent to which insomnia contributes to lifetime risk of CVD and the effects of sleep promotion beginning early in life.

- Differentiate the contributions of insomnia severity and specific sleep characteristics (e.g., duration, efficiency, timing) to CVD risk.

- Examine the effects of CBT-I in cardiovascular patients who have comorbid sleep disordered breathing.

- Evaluate the effectiveness of a CBT-I in CVD settings, including

"stepped-care" approaches and variations in frequency, duration, mode of delivery, and therapist preparation in cardiovascular care and cardiopulmonary rehabilitation settings. dimensional nature of sleep-related symptoms in everyday life.

CBT-I-related improvement of daytime symptoms extends previous cross-sectional findings [8] by supporting the causal relationship between insomnia and fatigue - a finding that contrasts with the common perception that fatigue is solely attributable to HF itself. The association between insomnia improvement and decreases in anxiety and depression is consistent with previous studies suggesting that sustained remission from depression is dependent on addressing insomnia [68]. The direct effects on anxiety and depression are consistent with studies of the effects of CBT focused on depression and exercise among patients with HF [69] and among patients with HF and COPD [70] and suggest that CBT-I effects may generalize to mood. The extent to which the improvements in anxiety or depression are dependent on improvements in sleep and insomnia merits examination in a larger RCT.

\section{Age and Gender Composition of CVD Groups}

Among the studies reviewed in this paper, the primary population focus was people 55 years of age and older - a group that often already suffers the age-related effects of CVD. Future studies are needed that consider the extent to which CBT-I prevents CVD or contributes to improvement in biomarkers of CVD risk in a broader age range of participants, including younger adults and children. Although age is often a contributor to insomnia and decreased sleep efficiency, insomnia was not associated with age in HF patients [8] and did not contribute to CBT-I outcomes in this population [36••]. Studies that consider the role of gender may be particularly important given the often-documented higher prevalence of insomnia in women.

\section{Insomnia Measurement}

Although there is a need for standard approaches to insomnia measurement in research [71], diagnostic nosologies and associated measures of sleep characteristics have varied (e.g., DSM [63], International Classification of Sleep Disorders [64]) in CBT-I trials and make results difficult to compare or generalize. Studies with self-reports of sleep quality have not always included specific quantitative measure of sleep attributes (e.g., sleep duration, latency, fragmentation, psychophysiological arousal, day-day variability in sleep) that often accompany insomnia. Understanding the role of specific sleep characteristics is important because of possible differential effects of sleep duration [18, 72, 73] and insomnia-related arousal [21], on inflammatory and metabolic effects and morbidity and mortality [73] and may be obscured by global or imprecise measures. Comparability of insomnia metrics may 
improve with recent efforts to harmonize the International Classification of Sleep Disorders (ICSD) and DSM-V nosologies and re-categorization of insomnia types.

Research is also needed to need for research to further specify the sleep attributes (or combination of attributes) that contribute to CVD outcomes and to identify the critical specific components of CBT-I that contribute to these outcomes. For example, the studies by Irwin and colleagues [48••, 49•, 50] are notable in their focus on inflammatory biomarkers related to insomnia, but limitations include global measure of insomnia without considering the possible differential of specific characteristics such as sleep duration, arousal, and circadian rhythmicity. Different components of CBT-I may support sleep extension, regularize sleep patterns, or decrease psychophysiological arousal, and the relevance of these components may depend on the sleep attributes of most relevance to CVD outcomes.

\section{Sleep Disordered Breathing and Insomnia}

Sleep disordered breathing (SDB), including obstructive and central sleep apnea, is often comorbid with insomnia, especially among people who are at risk for or already have CVD. Although SDB did not explain symptoms and functional performance in stable HF patients [57], these conditions are wellknown contributors to CVD morbidity and mortality, sleep deprivation, and biomarkers of cardiovascular risk. Although some CBT-I studies have been limited to patients without or mild SDB in order to control its possible confounding effects, this practice ignores the high prevalence of these conditions in CVD and the potential continued presence of insomnia even when patients are adherent to positive airway pressure treatment [74]. On the other hand, exclusion of people with SDB in CBT-I studies does not adequately represent the presence of these comorbid conditions and excludes a potentially large group who are at risk for both conditions. Future studies should address comorbidity of these sleep disorders.

\section{Screening and Access to Sleep Disorders Treatment}

The importance of sleep disorders to CVD suggests the need for screening and evaluation for the presence of sleep disorders, including insomnia and sleep-related symptoms. Failure of non-sleep specialist providers to evaluate sleep complaints has been well documented in a variety of populations and settings, and a recent study of HF patients revealed patients' dismay that health care providers fail to address insomnia [42]. Although there is a need for further study of the specific therapeutic effects of CBT-I relative to specific CVD outcomes, CBT-I is the first-line treatment for insomnia [75] and patients with evidence of this condition should be referred for treatment.

Access to CBT-I is an important problem in sleep medicine, but improved awareness among primary care and other health care providers, including cardiology providers, about the need to assess for the presence of sleep disorders likely increases access by improving the likelihood of treatment. However, knowledge of the best ways to assess for the presence of sleep disorders, discern possible risk for insomnia vs. SDB, and identify the need for referral for specialized sleep evaluation is often limited among patients and health care providers who are not sleep specialists. Anecdotal reports suggest that patients do not understand the distinction between insomnia and SDB, even when they have a prescription for positive airway pressure therapy (PAP) and may seek insomnia treatment in lieu of adherence to PAP [36••]. Hypnotic medications may also be inappropriately prescribed for patients who have undetected SDB $\left[36 \bullet^{\circ}\right]$ and are associated with important daytime adverse consequences, especially in older adults. Education of CVD patients and providers is needed to overcome these problems.

Access to CBT-I is limited by the absence of trained providers [76, 77], and the availability of trained master's degree prepared psychology professionals [77], registered nurses [54] and advanced practice nurses [55] and/or use of technology may extend access to CBT-I to primary care and cardiology settings. If larger randomized controlled trials document the sustained effects of CBT-I in HF and other CVD populations, CBT-I may become an important component of cardiac rehabilitation programs or HF self-management education [52], but strategies to assure effectiveness, including effective reach, implementation, and sustainability, will be needed and should be tested prior to full-scale implementation. Integration of behavioral services into chronic disease management programs is acceptable to patients and may also decrease health care resource utilization [78]. The wide variety of approaches to CBT-I components (e.g., relaxation vs. no relaxation), delivery methods (e.g., face-face, internet, video, face-face, group, self-help, therapist-directed), supportive materials (e.g., self-help booklets, video, sleep diaries), frequency and duration of the sessions, and the disciplinary training of the therapist suggest the importance of understanding the contributions of these variations to expected outcomes. A stepped care approach, such as recommended by Espie [76], may be especially useful in cardiovascular settings, but its role should be established when providing sleep care to CVD patients.

\section{Conclusion}

CBT-I shows promise as a way to reduce cardiovascular morbidity and symptom burden and increase functional performance and quality of life in the large population of people 
who are living with chronic CVD and insomnia. Additional research is needed to answer basic questions about short- and long-term outcomes, the role of biomarkers, and optimal delivery methods of CBT-I in cardiac conditions. In the meantime, assessment for the presence of sleep problems, including insomnia and appropriate referral for CBT-I, the first line treatment for this condition, is needed.

Acknowledgments This work was funded by P20NR014126, R21NR011387, and NR008022 (N. Redeker, PI) and 2T32NR008346 (N. Reynolds, PI).

\section{Compliance with Ethics Guidelines}

Conflict of Interest The authors declare that they have no competing interests.

Human and Animal Rights and Informed Consent This article does not contain any studies with human or animal subjects performed by any of the authors.

\section{References}

Papers of particular interest, published recently, have been highlighted as:

- Of importance

•- Of major importance

1. World Health Organization. The top 10 causes of death. 2014.

2. Morin CM, Benca R. Chronic insomnia. Lancet. 2012;379(9821): 1129-41.

3. LeBlanc M, Merette C, Savard J, Ivers H, Baillargeon L, Morin $\mathrm{CM}$. Incidence and risk factors of insomnia in a population-based sample. Sleep. 2009;32(8):1027-37.

4. Morin CM, LeBlanc M, Daley M, Gregoire JP, Merette C. Epidemiology of insomnia: prevalence, self-help treatments, consultations, and determinants of help-seeking behaviors. Sleep Med. 2006; 7(2):123-30

5. Budhiraja R, Roth T, Hudgel DW, Budhiraja P, Drake CL. Prevalence and polysomnographic correlates of insomnia comorbid with medical disorders. Sleep. 2011;34(7):859-67.

6. Taylor DJ, Mallory LJ, Lichstein KL, Durrence HH, Riedel BW, Bush AJ. Comorbidity of chronic insomnia with medical problems. Sleep. 2007;30(2):213-8.

7. Coryell VT, Ziegelstein RC, Hirt K, Quain A, Marine JE, Smith MT. Clinical correlates of insomnia in patients with acute coronary syndrome. Int Heart J. 2013;54(5):258-65.

8. Redeker NS, Jeon S, Muench U, Campbell D, Walsleben J, Rapoport DM. Insomnia symptoms and daytime function in stable heart failure. Sleep. 2010;33(9):1210-6.

9. Bonnet MH, Arand DL. Hyperarousal and insomnia: state of the science. Sleep Med Rev. 2010;14(1):9-15.

10. Farina B, Dittoni S, Colicchio S, et al. Heart rate and heart rate variability modification in chronic insomnia patients. Behav Sleep Med. 2014;12(4):290-306.

11. Canivet C, Nilsson PM, Lindeberg SI, Karasek R, Ostergren PO. Insomnia increases risk for cardiovascular events in women and in men with low socioeconomic status: a longitudinal, register-based study. J Psychosom Res. 2014;76(4):292-9.
12. Li M, Zhang XW, Hou WS, Tang ZY. Insomnia and risk of cardiovascular disease: a meta-analysis of cohort studies. Int J Cardiol. 2014;176(3):1044-7.

13. Silva-Costa A, Griep RH, Rotenberg L. Disentangling the effects of insomnia and night work on cardiovascular diseases: a study in nursing professionals. Braz J Med Biol Res $=$ Revista brasileira de pesquisas medicas e biologicas / Sociedade Brasileira de Biofisica ... [et al.]. 2015;48(2):120-127.

14. Parthasarathy S, Vasquez MM, Halonen M, et al. Persistent insomnia is associated with mortality risk. Am J Med. 2015;128(3):268275 e262.

15. Spiegelhalder K, Scholtes C, Riemann D. The association between insomnia and cardiovascular diseases. Nat Sci Sleep. 2010;2:71-8.

16. Phillips B, Buzkova P, Enright P. Insomnia did not predict incident hypertension in older adults in the cardiovascular health study. Sleep. 2009;32(1):65-72.

17. Laugsand LE, Vatten LJ, Bjorngaard JH, Hveem K, Janszky I. Insomnia and high-sensitivity C-reactive protein: the HUNT study, Norway. Psychosom Med. 2012;74(5):543-53.

18. Vgontzas AN, Liao D, Bixler EO, Chrousos GP, Vela-Bueno A. Insomnia with objective short sleep duration is associated with a high risk for hypertension. Sleep. 2009;32(4):491-7.

19. Pepin JL, Borel AL, Tamisier R, Baguet JP, Levy P, Dauvilliers Y. Hypertension and sleep: overview of a tight relationship. Sleep Med Rev. 2014;18(6):509-19.

20. Gottlieb DJ, Redline S, Nieto FJ, et al. Association of usual sleep duration with hypertension: the Sleep Heart Health Study. Sleep. 2006;29(8):1009-14.

21. Li Y, Vgontzas AN, Fernandez-Mendoza J, et al. Insomnia with physiological hyperarousal is associated with hypertension. Hypertension. 2015;65(3):644-50.

22. Haaramo P, Rahkonen O, Hublin C, Laatikainen T, Lahelma E, Lallukka T. Insomnia symptoms and subsequent cardiovascular medication: a register-linked follow-up study among middle-aged employees. J Sleep Res. 2014;23(3):281-9.

23. Sands-Lincoln M, Loucks EB, Lu B, et al. Sleep duration, insomnia, and coronary heart disease among postmenopausal women in the Women's Health Initiative. J Womens Health. 2013;22(6):477-86.

24. Rutledge T, Reis SE, Olson M, et al. Depression is associated with cardiac symptoms, mortality risk, and hospitalization among women with suspected coronary disease: the NHLBI-sponsored WISE study. Psychosom Med. 2006;68(2):217-23.

25. Laugsand LE, Vatten LJ, Platou C, Janszky I. Insomnia and the risk of acute myocardial infarction: a population study. Circulation. 2011;124(19):2073-81

26. Laugsand LE, Strand LB, Platou C, Vatten LJ, Janszky I. Insomnia and the risk of incident heart failure: a population study. Eur Heart J. 2014;35(21):1382-93.

27. Sofi F, Cesari F, Casini A, Macchi C, Abbate R, Gensini GF. Insomnia and risk of cardiovascular disease: a meta-analysis. Eur J Prev Cardiol. 2014;21(1):57-64.

28. Phillips B, Mannino D. Correlates of sleep complaints in adults: the ARIC study. J Clin Sleep Med: JCSM: Off Publ Am Acad Sleep Med. 2005;1(3):277-83.

29. Conley S, Feder S, Redeker NS. The relationship between pain, fatigue, depression and functional performance in stable heart failure. Heart Lung. 2015;44(2):107-12.

30. Gau FY, Chen XP, Wu HY, Lin ML, Chao YF. Sleep-related predictors of quality of life in the elderly versus younger heart failure patients: a questionnaire survey. Int J Nurs Stud. 2011;48(4): 419-28

31. Lee KS, Song EK, Lennie TA, et al. Symptom clusters in men and women with heart failure and their impact on cardiac event-free survival. J Cardiovasc Nurs. 2010;25(4):263-72. 
32. Song EK, Moser DK, Rayens MK, Lennie TA. Symptom clusters predict event-free survival in patients with heart failure. J Cardiovasc Nurs. 2010;25(4):284-91.

33. Banack HR, Holly CD, Lowensteyn I, et al. The association between sleep disturbance, depressive symptoms, and health-related quality of life among cardiac rehabilitation participants. J Cardiopulm Rehabil Prev. 2014;34(3):188-94.

34. Baldwin C, Griffith KA, Nieto J, O'Connor GT, Walsleben JA, Redline S. The association of sleep-disordered breathing and sleep symptoms with quality of life in the Sleep Heart Health Study. Sleep. 2001;24(1):96-105.

35. Redeker NS, Hilkert R. Sleep and quality of life in stable heart failure. J Card Fail. 2005;11(9):700-4.

36.• Redeker NS, Jeon S, Cline JC, Jacoby D, Mohensin V. Feasibility, acceptability and efficacy of cognitive behavioral therapy for insomnia in stable heart failure. J Clin Sleep Med. 2015 (in press). Researchers randomized 48 participants with stable heart failure and insomnia into two groups: CBT-I group or sleep education and heart failure self-management group. CBT-I had a significant effect on insomnia and fatigue when controlling for age and comorbidity.

37. Sivertsen B, Lallukka T, Salo P, et al. Insomnia as a risk factor for ill health: results from the large population-based prospective HUNT Study in Norway. J Sleep Res. 2014;23(2):124-32.

38. Morin CM, Bootzin RR, Buysse DJ, Edinger JD, Espie CA, Lichstein KL. Psychological and behavioral treatment of insomnia: update of the recent evidence (1998-2004). Sleep. 2006;29(11): $1398-414$

39. Morin CM. Insomnia: psychological assessment and management. New York: Guilford Press; 1993.

40. Espie CA. The psychological treatment of insomnia. Chichester: Wiley; 1991.

41. Morin CM. Psychological and behavioral treatments for primary insomnia. In: Kryger MH, Roth T, Dements WC, editors. Principles and practice of sleep medicine. 4th ed. Philadelphia: Elsevier Saunders; 2005.

42. Andrews LK, Coviello J, Hurley E, Rose L, Redeker NS. "I'd eat a bucket of nails if you told me it would help me sleep:" perceptions of insomnia and its treatment in patients with stable heart failure. Heart Lung. 2013;42(5):339-45.

43. Bluestein D, Healey AC, Rutledge CM. Acceptability of behavioral treatments for insomnia. J Am Board Fam Med. 2011;24(3): 272-80.

44. Rybarczyk B, Lopez M, Benson R, Alsten C, Stepanski E. Efficacy of two behavioral treatment programs for comorbid geriatric insomnia. Psychol Aging. 2002;17(2):288-98.

45.• Rybarczyk B, Mack L, Harris JH, Stepanski E. Testing two types of self-help CBT-I for insomnia in older adults with arthritis or coronary artery disease. Rehabil Psychol. 2011;56(4):257-66. Researchers randomized 106 older adults (age $\geq 55$ years) with insomnia and osteoarthritis, coronary artery disease or no major medical condition into two groups: a book version and a multimedia enhanced CBT-I. The researchers found both versions of CBT-I were efficacious at improving sleep posttreatment and that there was no significance difference between no major medical conditions, arthritis or coronary artery disease.

46. Rybarczyk B, Stepanski E, Fogg L, Lopez M, Barry P, Davis A. A placebo-controlled test of cognitive-behavioral therapy for comorbid insomnia in older adults. J Consult Clin Psychol. 2005;73(6): 1164-74.

47. Riemann D, Perlis ML. The treatments of chronic insomnia: a review of benzodiazepine receptor agonists and psychological and behavioral therapies. Sleep Med Rev. 2009;13(3):205-14.

48.• Irwin MR, Olmstead R, Carrillo C, et al. Cognitive behavioral therapy vs. Tai Chi for late life insomnia and inflammatory risk: a randomized controlled comparative efficacy trial. Sleep. 2014;37(9):1543-52. Researchers randomized 123 older adults (age $>55$ years) with insomnia into three groups; CBT-I, Thai Chi Chih (TCC), and a sleep seminar education control. CBT performed better than the TCC or sleep seminar to induce remission of insomnia. At 16 months CBT-I compared to sleep seminar had reduced risk of high CRP $(>3.0 \mathrm{mg} / \mathrm{ml})$.

49. Irwin MR, Olmstead R, Breen EC, et al. Cognitive behavioral therapy and Tai Chi reverse cellular and genomic markers of inflammation in late life insomnia: a randomized controlled trial. Biol Psychiatry. 2015. doi: 10.1016/j. biopsych.2015.01.010. Researchers randomized 123 older adults (age $>55$ years) with insomnia into three groups; CBT-I, Thai Chi Chih (TCC), and a sleep seminar education control. CBT-I significantly reduced CRP at 4 and 16 months. TCC significantly reduced monocyte production of proinflammatory cytokines at months 2,4 , 7, and 16 and proinflammatory gene expression at month 4.

50. Carroll JE, Seeman TE, Olmstead R, et al. Improved sleep quality in older adults with insomnia reduces biomarkers of disease risk: pilot results from a randomized controlled comparative efficacy trial. Psychoneuroendocrinology. 2015;55:184-92.

51. Redeker NS, Jeon S, Andrews L, Cline J, Pacelli J, Jacoby D. Cognitive behavioral therapy for insomnia has sustained effects on daytime symptoms and hospitalization in patients with stable heart failure. Sleep. 2013;36:A297.

52. Redeker NS, Jeon S, Pacelli J, Anderson G. Sleep disturbance, sleep-related symptoms, and biological rhythms in heart failure patients who have insomnia. Sleep. 2014;37:A248-9.

53. Redeker NS, Jeon S, Andrews L, Cline J, Jacoby D, Mohsenin V. Feasibility and efficacy of a self-management intervention for insomnia in stable heart failure. J Clin Sleep Med : JCSM : Off Publ Am Acad Sleep Med. 2015. CBT-I is feasible and acceptable and has short-term efficacy on insomnia and fatigue in $\mathrm{HF}$.

54. Espie CA, MacMahon KM, Kelly HL, et al. Randomized clinical effectiveness trial of nurse-administered small-group cognitive behavior therapy for persistent insomnia in general practice. Sleep. 2007;30(5):574-84.

55. Buysse DJ, Germain A, Moul DE, et al. Efficacy of brief behavioral treatment for chronic insomnia in older adults. Arch Intern Med. 2011;171(10):887-95.

56. Bastien $\mathrm{CH}$, Vallieres $\mathrm{A}$, Morin $\mathrm{CM}$. Validation of the Insomnia Severity Index as an outcome measure for insomnia research. Sleep Med. 2001;2(4):297-307.

57. Redeker NS, Muench U, Zucker MJ, et al. Sleep disordered breathing, daytime symptoms, and functional performance in stable heart failure. Sleep. 2010;33(4):551-60.

58. Dekker RL, Moser DK, Peden AR, Lennie TA. Cognitive therapy improves three-month outcomes in hospitalized patients with heart failure. J Card Fail. 2012;18(1):10-20.

59. Gulliksson M, Burell G, Vessby B, Lundin L, Toss H, Svardsudd K. Randomized controlled trial of cognitive behavioral therapy vs standard treatment to prevent recurrent cardiovascular events in patients with coronary heart disease: Secondary Prevention in Uppsala Primary Health Care project (SUPRIM). Arch Intern Med. 2011;171(2):134-40.

60. Irwin MR. Why sleep is important for health: a psychoneuroimmunology perspective. Annu Rev Psychol. 2015;66:143-72.

61. Cho HJ, Seeman TE, Kiefe CI, Lauderdale DS, Irwin MR. Sleep disturbance and longitudinal risk of inflammation: moderating influences of social integration and social isolation in the Coronary Artery Risk Development in Young Adults (CARDIA) study. Brain Behav Immun. 2015;46:319-26.

62. Vgontzas AN, Bixler EO, Lin H-M, et al. Chronic insomnia is associated with nyctohemeral activation of the hypothalamic- 
pituitary-adrenal axis: clinical implications. J Clin Endocrinol Metab. 2001;86(8):3787-94.

63. Association AP. In: Fourth TR, editor. Diagnostic and Statistical Manual of Mental Disorders. Arlington: American Psychiatric Association; 2000.

64. American Academy of Sleep Medicine. The international classification of sleep disorders. Westchester: American Academy of Sleep Medicine; 2005.

65. Savard J, Simard S, Ivers H, Morin CM. Randomized study on the efficacy of cognitive-behavioral therapy for insomnia secondary to breast cancer, part II: immunologic effects. J Clin Oncol. 2005;23(25):6097-106.

66. Vitiello MV, McCurry SM, Rybarczyk BD. The future of cognitive behavioral therapy for insomnia: what important research remains to be done? J Clin Psychol. 2013;69(10):1013-21.

67. Fleming L, Randell K, Harvey CJ, Espie CA. Does cognitive behaviour therapy for insomnia reduce clinical levels of fatigue, anxiety and depression in cancer patients? Psychooncology. 2014;23(6):679-84.

68. Manber R, Edinger JD, Gress JL, San Pedro-Salcedo MG, Kuo TF, Kalista T. Cognitive behavioral therapy for insomnia enhances depression outcome in patients with comorbid major depressive disorder and insomnia. Sleep. 2008;31(4):489-95.

69. Gary RA, Dunbar SB, Higgins MK, Musselman DL, Smith AL. Combined exercise and cognitive behavioral therapy improves outcomes in patients with heart failure. J Psychosom Res. 2010;69(2): $119-31$.

70. Cully JA, Stanley MA, Deswal A, Hanania NA, Phillips LL, Kunik ME. Cognitive-behavioral therapy for chronic cardiopulmonary conditions: preliminary outcomes from an open trial. Prim Care Companion J Clin Psychiatry. 2010;12(4):e1-6.

71. Buysse DJ, Ancoli-Israel S, Edinger JD, Lichstein KL, Morin CM. Recommendations for a standard research assessment of insomnia. Sleep. 2006;29(9):1155-73.

72. Vgontzas AN, Fernandez-Mendoza J, Liao D, Bixler EO. Insomnia with objective short sleep duration: the most biologically severe phenotype of the disorder. Sleep Med Rev. 2013;17(4):241-54.

73. Mullington JM, Haack M, Toth M, Serrador JM, Meier-Ewert HK. Cardiovascular, inflammatory, and metabolic consequences of sleep deprivation. Prog Cardiovasc Dis. 2009;51(4):294-302.

74. Ong JC, Crisostomo MI. The more the merrier? Working towards multidisciplinary management of obstructive sleep apnea and comorbid insomnia. J Clin Psychol. 2013;69(10):1066-77.

75. National Institutes of Health. National Institutes of Health State of the Science Conference Statement. Manifestations and management of chronic insomnia in adults. Sleep. 2005;28:1049-57.

76. Espie CA. "Stepped care": a health technology solution for delivering cognitive behavioral therapy as a first line insomnia treatment. Sleep. 2009;32(12):1549-58.

77. Fields BG, Schutte-Rodin S, Perlis ML, Myers M. Master's-level practitioners as cognitive behavioral therapy for insomnia providers: an underutilized resource. J Clin Sleep Med: JCSM: Off Publ Am Acad Sleep Med. 2013;9(10):1093-6.

78. Kinsinger SW, Ballou S, Keefer L. Snapshot of an integrated psychosocial gastroenterology service. World J Gastroenterol: WJG. 2015;21(6):1893-9. 